\title{
Isolation and Identification of Cultivable Microorganisms Isolated from Sea Squirt (Ciona savignyi) Collected from the Jiaozhou Bay, China
}

\section{Dandan Wang ${ }^{1,2}$, Chuanwei Li³, Yuxi Wei ${ }^{1}$}

\author{
${ }^{1}$ Life Science College, Qingdao University, Qingdao 266071, P. R. China \\ ${ }^{2}$ Li Key Laboratory of Marine Drugs, Ministry of Education, School of Medicine and Pharmacy, Ocean University \\ of China, Qingdao 266003, P. R. China \\ ${ }^{3}$ College of Marine Life Sciences, Ocean University of China, Qingdao 266003, P. R. China
}

\begin{abstract}
Sea squirt is an important source for isolation of new bacteria strains that are able to accumulate bio-active compounds. In this study, we isolated and identified of cultivable bacteria isolated from sea squirt Ciona savignyi, collected from the Tsingtao Port, Jiaozhou Bay, China. We investigated the phenotypic characteristics and neighbour-joining phylogenetic analysis of this isolated cultivable strains, which suggested the isolated seventeen strains mainly belong to the Proteobacteria, Bacteroidetes and Actinobacteria four phyla, of which twelve strains belong to Proteobacteria, accounted for $70.6 \%$ of total isolated stains, and respectively belonging to Alphaproteobacteria and Gammaproteobacteria. This identification and analysis of this these marine bacteria will enrich sea squirt-associated bacteria resources, from which more bio-active substances would be discovered.
\end{abstract}

Keywords: Cultivable Microorganisms, Phylogenetic Analysis, Marine Bacteria, Sea Squirt, Isolation And Identification

\section{Introduction}

Marine microorganism is widely distributed, there are some strains living in the sea, some existing on the surface of the sea and some living in sediment or on the surface of mud, while some have the codependent, symbiotic and parasitic or associated relationship with marine animals or plants. Associated bacteria may play an significant role in clearing metabolic waste of the host (Wikjinson C R , 1978) and providing the bio-active substances for the host (Unson M. D. et al.; Schmidt E. W. et al.; Hentschel U. et al.). Because of co-evolution of the associated bacteria with the host for a long time, the associated bacteria often have special metabolic pathway, it is likely to produce new physiological active substances. Therefore many researchers have the considerable interests in the using of the associated marine microorganisms as a source of the natural biological products (Oern P.). Microorganisms have the advantages of the short growth cycle, easy controlling metabolism and easy breeding of strains, therefore, there is wide availability of using and development of the associated microorganism resources. The isolation of bio-active substances from

This article is published under the terms of the Creative Commons Attribution License 4.0

Author(s) retain the copyright of this article. Publication rights with Alkhaer Publications.

Published at: http://www.ijsciences.com/pub/issue/2017-03/

DOI: 10.18483/ijSci.1216; Online ISSN: 2305-3925; Print ISSN: 2410-4477 
associated microorganisms not only protects the environment but also reduces the cost of the commercial production.

There are lots of marine microorganisms living on the surface and in the coelom of the marine invertebrates, and they have highly specific associated relations with the marine invertebrates. At present, the researches of marine invertebrates are mainly concentrated on the sponge, sea anemones, corals and some coelenterate. In recent years, many researchers also found a large amount of novel structure and unique active compounds in the sea squirt (Ryuichi $\mathrm{S}$ et al.; Mcdonald L. A. et al.; Williams A. B et al.). These novel active compounds have attracted many researchers' attention, and have gradually become hot research on marine natural products chemistry, sea squirts became the important marine biological resources to obtain significant physiological active substances for human. Because of many marine biological active compounds is produced by the associated marine microorganisms, thus isolated associated-sea squirts microbial resources and studied its phylogenetic diversity, will be able to provide new data for marine bio-active compounds.

This study we isolated and identified of cultivable bacteria isolated from sea squirt Ciona savignyi, we investigated the phenotypic and phylogenetic characteristics and neighbour-joining phylogenetic analysis of the isolated strains. Therefore, the identification of marine bacteria will provide the basis for further exploring more bio-active substances and marine biological community distribution.

\section{Materials and Methods}

\section{Experimental sample}

A sea squirt (C. savignyi) was collected by scuba diver in the Tsingtao Port $\left(36^{\circ} 04^{\prime} 00^{\prime \prime} \mathrm{N}, 120^{\circ} 19^{\prime} 05^{\prime \prime} \mathrm{E}\right)$, Jiaozhou Bay, China, in May 2015, and was used as the source for isolation of squirt-associated bacteria.

\section{The separation and purification of strains}

The inner content of the sea squirt was serially diluted using $0.9 \%(\mathrm{w} / \mathrm{v}) \mathrm{NaCl}$, and then were spread on marine 2216E agar (per litre seawater: $5 \mathrm{~g}$ peptone, $1 \mathrm{~g}$, yeast extract, $0.1 \mathrm{~g}$ ferric phosphate, $15 \mathrm{~g}$ agar; $\mathrm{pH}$ 7.6-7.8). The cultivable strains were isolated after 10 days of incubation and was cultivated routinely on MA at $28{ }^{\circ} \mathrm{C}$. Culture purity was confirmed by homogeneity of the cell and colony morphologies and then observed and recorded the colony characteristics of the marine bacteria isolated from Ciona savignyi. Stored the isolated strains at $-80{ }^{\circ} \mathrm{C}$ supplemented with $50 \%$ (v/v) glycerol.

\section{The 16S rRNA gene sequences alignment}

For DNA extraction, cell biomass of strains was obtained from cultures grown in marine broth 2216 (per litre seawater: $5 \mathrm{~g}$ peptone, $1 \mathrm{~g}$,yeast extract, 0.1 $\mathrm{g}$ ferric phosphate; $\mathrm{pH} 7.6-7.8)$ at $28{ }^{\circ} \mathrm{C}$ for $24 \mathrm{~h}$. Chromosomal DNA was extracted and purified according to standard methods (Ausubel et al., 1995). The 16S rRNA gene was amplified by PCR with two universal primers (27F,5'-AGAGTTTGATCCTGGCTCAG-3' and 1492R, 5'-GGTTACCTTGTTACGACTT-3'). The components and condition of PCR reaction see Table 1, 2. After purification using an E.Z.N.A gel extraction kit (OMEGA Biotech), the amplified 16S rRNA gene was ligated into the pMD19-T (TaKaRa Clontech), which was further sequenced at TsingKe Biotech (Qingdao, China). Sequence homology values between the 16S rRNA gene from isolated strains and closely related type strains were analysed using the EzTaxon-e server (http://eztaxon-e. ezbiocloud.net/; Kim et al., 2012). 
Table 1 Components of PCR reaction

\begin{tabular}{ll}
\hline Components & Volume $(\mu \mathrm{l})$ \\
\hline $2 \times$ taq PCR MasterMix $($ TIANGEN & 50 \\
Biotech Beijing) & \\
Primer 27F & 5 \\
Primer1492R & 5 \\
DNA & 5 \\
ddH $_{2} \mathrm{O}$ & 35 \\
total & 100 \\
\hline
\end{tabular}

Table 2 Condition of PCR reaction

\begin{tabular}{|c|c|}
\hline Reaction & Condition \\
\hline Pre-Denaturation & $95^{\circ} \mathrm{C} 5 \mathrm{~min}$ \\
\hline Denaturation & $95^{\circ} \mathrm{C} 30 \mathrm{~s}$ \\
\hline Annealing & 25 cycles \\
\hline Extension & $72{ }^{\circ} \mathrm{C} 90 \mathrm{~s}$ \\
\hline Post-Extension & $72{ }^{\circ} \mathrm{C} 5 \mathrm{~min}$ \\
\hline
\end{tabular}

Neighbour-joining phylogenetic analysis based on 16S rRNA gene sequence

Multiple alignments of the 16S rRNA gene sequence of isolated strains with those of its related strains were performed using the CLUSTAL_X (Thompson et al., 1997). Phylogenetic trees were constructed by the neighbour-joining (N-J) (Saitou \& Nei, 1987), algorithms using MEGA 6.06 (Tamura et al., 2013), and were analyzed using bootstrapping (Felsenstein, 1985) based on 1000 re-samplings.

\section{Results and Discussion}

Morphology characteristics of the isolated strains

We obtained cultivable bacteria isolated from sea squirt (Ciona savignyi). They have various morphology characteristics (Table 3) and was distinguished by color shape, height, uniformity, surface, humidity, transparency. Most of the stains were roundness, dimpling, orderliness, smooth, humid and opacity.

Table 3 The colony characteristics of the marine bacteria isolated from Ciona savignyi

\begin{tabular}{llllllll}
\hline NO. & Color & Shape & \multicolumn{1}{c}{ Height } & Uniformity & Surface & Humidity & Transparency \\
\hline H-1 & Cream white & Roundness & Embossment & Orderliness & Smooth & Humid & Opacity \\
H-3 & Cream white & Roundness & Embossment & Orderliness & Smooth & Wet & Opacity \\
H-4 & Orange & Roundness & Dimpling & Orderliness & Smooth & Humid & Opacity \\
H-6 & Yellow & Roundness & Dimpling & Orderliness & Level & Humid & Opacity \\
H-7 & Orange & Random & Embossment & Irregular & Smooth & Humid & Opacity \\
H-8 & White & Roundness & Flat & Irregular & Unfairness & Middle & Translucent \\
H-9 & White & Random & Flat & Irregular & Level & Middle & Opacity \\
H-10 & brownish & Oval & Blowup & Orderliness & Smooth & Wet & Opacity \\
H-11 & Saffron & Roundness & Embossment & Orderliness & Smooth & Humid & Opacity \\
& yellow & & & & & & \\
H-12 & Yellowish & Roundness & Embossment & Orderliness & Smooth & Humid & Opacity \\
& white & & & & & & \\
H-15 & Cream white & Roundness & Dimpling & Orderliness & Smooth & Wet & Opacity \\
H-16 & Gray & Roundness & Flat & Irregular & Unfairness & Dry & Opacity \\
H-17 & Yellowish & Roundness & Dimpling & Orderliness & Level & Middle & Opacity \\
& white & & & & & & \\
H-18 & Roundness & Roundness & Blowup & Orderliness & Smooth & Humid & Opacity \\
H-19 & White & Oval & Embossment & Orderliness & Smooth & Humid & Opacity \\
H-20 & Saffron & Roundness & Embossment & Orderliness & Smooth & Humid & Opacity
\end{tabular}


Isolation and Identification of Cultivable Microorganisms Isolated from Sea Squirt (Ciona savignyi) Collected from the Jiaozhou Bay, China

yellow

H-22 Saffron Roundness Embossment Orderliness Smooth Wet Opacity yellow

\section{The 16S rRNA gene sequences alignment}

We obtained seventeen cultivable bacteria isolated from Ciona savignyi. The agarose gel electrophoresis results of PCR products of $16 \mathrm{~S}$ rRNA gene as shown in Figure 1, gene fragment size is about 1500 b. The results of 16S rRNA gene sequences alignment of the isolated cultivable stains see the Table 4.

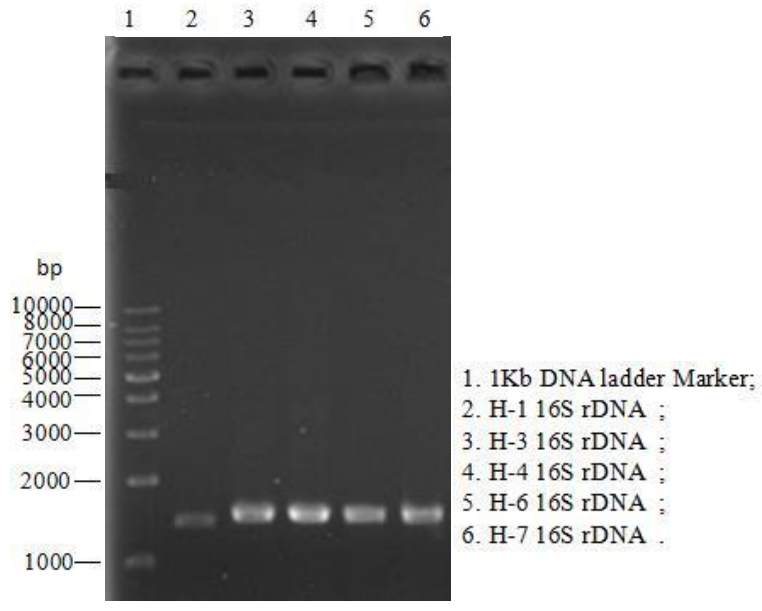

Figure 1 The agarose gel electrophoresis results of PCR products of $16 \mathrm{~S}$ rRNA gene

Table 4 The 16S rRNA gene sequences alignment results of the cultivable bacteria isolated from Ciona savignyi

\begin{tabular}{|c|c|c|c|}
\hline Phylogenetic groups & NO. & Closest type strain & Similarity $(\%)$ \\
\hline Proteobacteria & H-9 & Sulfitobacter pontiacus DSM $10014^{\mathrm{T}}$ & 99.78 \\
\hline \multirow[t]{4}{*}{ Alphaproteobacteria } & $\mathrm{H}-10$ & Phaeobacter inhibens DSM $16374^{\mathrm{T}}$ & 99.93 \\
\hline & H-12 & Amylibacter marinus $2-3^{\mathrm{T}}$ & 95.33 \\
\hline & $\mathrm{H}-25$ & Pseudophaeobacter arcticus DSM $23566^{\mathrm{T}}$ & 99.21 \\
\hline & H-18 & Celeribacter halophilus $\mathrm{ZXM} 137^{\mathrm{T}}$ & 98.82 \\
\hline Proteobacteria & $\mathrm{H}-1$ & Pseudoalteromonas marina $\mathrm{Mano}^{\mathrm{T}}$ & 99.71 \\
\hline \multirow[t]{6}{*}{ Gammaproteobacteria } & $\mathrm{H}-3$ & Vibrio atlanticus $\mathrm{Vb} 11.11^{\mathrm{T}}$ & 99.93 \\
\hline & $\mathrm{H}-8$ & Vibrio lentus $4 \mathrm{OM} 4^{\mathrm{T}}$ & 99.39 \\
\hline & $\mathrm{H}-11$ & Pseudoalteromonas tetraodonis IAM $14160^{\mathrm{T}}$ & 99.38 \\
\hline & H-19 & Marinomonas aquimarina CECT $5080^{\mathrm{T}}$ & 98.22 \\
\hline & $\mathrm{H}-20$ & Psychrosphaera saromensis SA4- $48^{\mathrm{T}}$ & 98.48 \\
\hline & $\mathrm{H}-22$ & Shewanella piezotolerans $\mathrm{WP}^{\mathrm{T}}$ & 99.67 \\
\hline Bacteroidetes; & $\mathrm{H}-4$ & Polaribacter reichenbachii $6 \mathrm{Alg} 8^{\mathrm{T}}$ & 98.06 \\
\hline \multirow[t]{2}{*}{ Flavobacteria } & H-6 & Tenacibaculum soleae LL04 $12.1 .7^{\mathrm{T}}$ & 99.05 \\
\hline & $\mathrm{H}-7$ & Winogradskyella crassostreae TYO-19 & 100.00 \\
\hline Actinobacteria; & H-16 & Streptomyces harbinensis NEAU-Da3 ${ }^{\mathrm{T}}$ & 99.72 \\
\hline Actinobacteria_c; & $\mathrm{H}-17$ & Kocuria palustris DSM $11925^{\mathrm{T}}$ & 100.00 \\
\hline
\end{tabular}

Neighbor-joining phylogenetic analysis based on 16S rRNA gene sequences

The isolated seventeen strains mainly belong to the
Proteobacteria, Bacteroidetes and Actinobacteria four phyla, of which twelve strains belong to Proteobacteria, accounted for $70.6 \%$ of total isolated 
Isolation and Identification of Cultivable Microorganisms Isolated from Sea Squirt (Ciona savignyi) Collected from the Jiaozhou Bay, China

stains, and respectively belonging to amount and type, while there are some important Alphaproteobacteria and Gammaproteobacteria species have been found in Gammaproteobacteria (Figure 2). Proteobacteria is one of the largest groups of bacteria (Bergey, 2003), and this is according to our study. In previous study found that such as genus Pseudomonas and Vibrios. In addition, we also isolated strains within the class Flavobacteria and Actinobacteria_c.

Alphaproteobacteria have the advantage on the

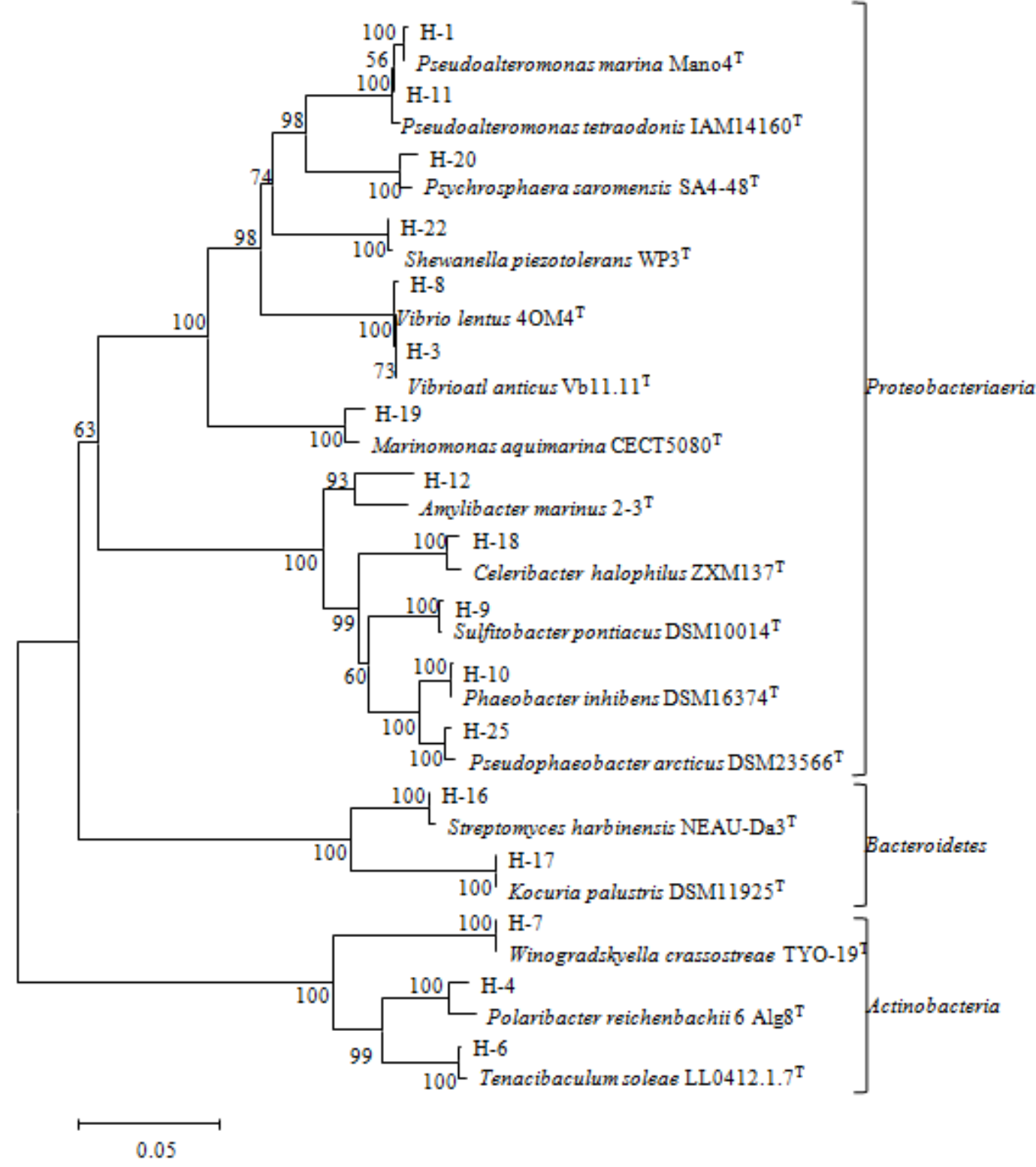

Figure 2 Neighbor-joining phylogenetic tree based on 16S rRNA gene sequences of the cultivable bacteria isolated from Ciona savignyi

\section{Conclusion}

This study we isolated and identified of cultivable bacteria isolated from sea squirt Ciona savignyi, collected from the Tsingtao Port, Jiaozhou Bay, China.
We investigated the phenotypic and phylogenetic characteristics and neighbour-joining phylogenetic analysis of this isolated seventeen cultivable strains, which suggested the isolated seventeen strains mainly 

the Jiaozhou Bay, China

belong to the Proteobacteria, Bacteroidetes and Actinobacteria four phyla, of which twelve strains belong to Proteobacteria, accounted for $70.6 \%$ of total isolated stains, and respectively belonging to Alphaproteobacteria and Gammaproteobacteria. The identification of marine bacteria will provide the basis for further studying the marine biological active compounds and exploring marine biological community distribution.

\section{Acknowledgment}

We would like to thank Professor Dong Bo (College of Marine Life Sciences; Ocean University of China; China) for providing us sea squirt (Ciona savignyi).

\section{References}

1) Wikjinson C. R., 1978. Micobial associations in sponges. I Ecology,physiology and micobial populations of coral reef sponges. Marine biology, 49: 161-167.

2) Unson M. D.,Holland N. D., Fqulkner D. J, 1994. A brominated secondry metabolite syntheaized by the cyanobacterial symbiont of a marine sponge and accumulation of the crystalline metabolite in the sponge tissue. Marine Biology, 119: 1-11

3) Schmidt E. W., Obraztsova A. Y., Davidson D. S. K., et al., 2000. Identification of the antifungal peptide-containing sybiont of the marine sponge Theonella swinhoei as a novel $\delta$-teobacterium, "Candidatus Entotheonella palauensis". Marine Biology, 136: 969-977.

4) Hentschel U., Schmid M., Wanger M. et al., 2001. Isolation and phyogenetic analysis of bacteria withe antimicribial activities from the Mediterranean sponges Apiysina aerphoba and Aplysina cavernicola. FEMS Microbiology Ecology, 35:
305-312.

5) Oern P., 2004. Metabolites from symbiotic bacteria. Nat. Prod. Rep., 21: 519-538.

6) Ryuichi S., Stroh J. G., Sullin D. W., et al., 1995. Seven new didemnins from the marine tunicate Tirdidemnum solidum. Journal of the Amerincan Chemical Society, 117: 3734-3736.

7) Mcdonald L. A., Swersey J. C., Ireland C. M., et al., 1995. Botryllamide A-D, new brominated tyrosine derivatives from styelid ascidians of the genus Botryllus. Tetrahedron, 51: 5237-5240.

8) Williams A. B., Jacobs R. S., 1993. A marine natural product, patellamide $\mathrm{D}$, reverses multidrud resistance in a human leukemic cell line. Cancer Letter, 71: 97-102.

9) Ausubel, F. M., Brent, R., Kingston, R. E., Moore, D. D., Seidman, J. G., Smith, J. A. \& Struhl, K. (editors), 1995. Short Protocols in Molecular Biology: a Compendium of Methods from Current Protocols in Molecular Biology, 3rd edn. New York: Wiley.

10) Kim, O. S., Cho, Y. J., Lee, K., Yoon, S. H., Kim, M., Na, H., Park, S. C., Jeon, Y. S., Lee, J. H. \& other authors , 2012. Introducing EzTaxon-e: a prokaryotic 16S rRNA gene sequence.

11) Thompson, J. D., Gibson, T. J., Plewniak, F., Jeanmougin, F. \& Higgins, D. G. , 1997. The CLUSTAL_X windows interface: flexible strategies for multiple sequence alignment aided by quality analysis tools. Nucleic Acids Res 25: 4876-4882.

12) Tamura, K., Stecher, G., Peterson, D., Filipski, A. \& Kumar, S. , 2013. MEGA6: molecular evolutionary genetics analysis version 6.0. Mol Biol Evol 30: 2725-2729.

13) Felsenstein, J. , 1985. Confidence limits on phylogenies: an approach using the bootstrap. Evolution 39: 783-791.

14) Munn C B , 2003 Marine Microbiology: Ecology and Applications. London and New York: BIOS Scientific Publishers.

15) Tong Yu, 2013. Cultivation of deep-sea microorganisms from the South China Sea and taxonomic analysis of four novel bacteria Master's degree thesis.Qingdao. 